\title{
Preparation of Higher Molecular Weight Poly (L-lactic Acid) by Chain Extension
}

\author{
Chenguang Liu, Yuliang Jia, and Aihua He \\ Key Laboratory of Rubber-Plastics, Ministry of Education/Shandong Provincial Key Laboratory of Rubber-Plastics, \\ Qingdao University of Science \& Technology, Qingdao 266042, China
}

Correspondence should be addressed to Aihua He; aihuahe@iccas.ac.cn

Received 7 June 2013; Accepted 18 July 2013

Academic Editor: Zhou Yang

Copyright (C) 2013 Chenguang Liu et al. This is an open access article distributed under the Creative Commons Attribution License, which permits unrestricted use, distribution, and reproduction in any medium, provided the original work is properly cited.

\begin{abstract}
High molecular weight poly (lactic acid) (PLA) was obtained by chain extending with hexamethylene diisocyanate (HDI). The influences of the amount of chain extender, reaction time, and molecular weight changes of prepolymers on the poly(lactic acid) were investigated. PLA prepolymer with a viscosity, average molecular weight $\left(M_{\eta}\right)$ of $2 \times 10^{4} \mathrm{~g} / \mathrm{mol}$ was synthesized from L-lactide using stannous octoate as the catalyst. After $20 \mathrm{~min}$ of chain extension at $175^{\circ} \mathrm{C}$, the resulting polymer had $M_{w}$ of $20.3 \times 10^{4} \mathrm{~g} / \mathrm{mol}$ and $M_{n}$ of $10.5 \times 10^{4} \mathrm{~g} / \mathrm{mol}$. Both FT-IR and ${ }^{1} \mathrm{H}$-NMR verified that the structure of PLA did not change either before chain extending or after. The optically active characterized that the chain extending-product was left handed. DSC and XRD results showed that both the $T_{g}$ and the crystallinity of PLA were lowered by chain-extension reaction. The crystalline transformation happened in PLA after chain extending, crystalline $\alpha^{\prime}$ form to $\alpha$ form.
\end{abstract}

\section{Introduction}

At present, plastics waste disposal has become a serious problem worldwide. There is a strong need to provide plastic materials suitable for packaging, which will also be degradable and result in products that are environmentally safe [1]. Concerning the feasibility of the use of renewable raw materials and production process, the poly (lactic acid) (PLA) has become a very promising biodegradable polymer $[2,3]$.

High molecular weights are needed for PLA to have good physical properties. Until now, high molecular weight PLA was synthesized by ring-opening polymerization of the lactide [4], which is relatively complicated and expensive. Direct polycondensation of lactic acid is a low-cost process to produce PLA [5]; however, it is hard to increase the molecular weight enough because of the difficulty of removing the water from the system. A large number of investigations have been made to improve PLA properties via plasticization, copolymerization, and blending with elastomers [614]. Among them, low-cost, nontoxic HDI as chain extender is the simplest and most effective way. Addition of a chain extender to improve the molecular weight of PLA, it can solve the problem of degradation time of polylactic acid being uncontrollable [15]. But most of the researches were focused on the molecular weight of the products, optical activities and the transition of crystal form of the products effect of chain extension reaction were ignored.

In this paper, higher molecular weight PLLA was produced by ring-opening polymerization of lactide followed by chain extension. Hexamethylene diisocyanate (HDI) was used as the chain extender. Optical activities and the transition of crystal form of the PLA chain extend with HDI were studied.

\section{Materials and Methods}

2.1. Materials. L-lactide was synthesized and purified by laboratory: $97 \%$ optically pure, $T_{m} 100.8^{\circ} \mathrm{C}$, and moisture content $19.0 \mathrm{ppm}$. Stannous octoate was from Sigma Chemical Co. HDI (+99\%) was purchased from Shanghai Jingchun Chemical Co., China. Toluene was refluxed over sodium with benzophenone as an indicator. All other chemicals were reagent grade. 
TABLE 1: The influences of process conditions on poly(lactic acid) after chain extension.

\begin{tabular}{|c|c|c|c|c|c|c|c|c|c|}
\hline \multirow{2}{*}{$\operatorname{Run}^{\mathrm{a}}$} & \multirow{2}{*}{$\begin{array}{c}M_{\eta \text { pre }} \\
\left(10^{-4} \mathrm{~g} / \mathrm{mol}\right)\end{array}$} & \multirow{2}{*}[\mathrm{NCO}]{$/[\mathrm{OH}]^{\mathrm{b}}$} & \multirow{2}{*}{$t(\min )$} & \multirow{2}{*}[\alpha]{$_{D}^{25}$} & \multicolumn{2}{|c|}{$M\left(10^{-4} \mathrm{~g} / \mathrm{mol}\right)$} & \multirow{2}{*}{$M_{w} / M_{n}$} & \multirow{2}{*}{$\begin{array}{c}\text { Chain-extension } \\
\text { efficiency }(\%)\end{array}$} & \multirow{2}{*}{$\begin{array}{l}\text { Insoluble content } \\
\text { (wt } \%)\end{array}$} \\
\hline & & & & & $M_{n}$ & $M_{w}$ & & & \\
\hline al & 2 & 1.5 & 20 & -141 & 7.9 & 16.3 & 2.0 & 68.9 & 2.2 \\
\hline $\mathrm{a} 2$ & 2 & 3 & 20 & -145 & 8.7 & 12.1 & 1.4 & 42.6 & 3.0 \\
\hline a3 & 2 & 6 & 20 & -145 & 10.5 & 20.3 & 1.9 & 52.2 & 3.6 \\
\hline a4 & 2 & 8 & 20 & -139 & 9.9 & 15.2 & 1.5 & 45.7 & 3.9 \\
\hline a5 & 2 & 6 & 10 & -140 & 5.4 & 8.3 & 1.6 & 47.9 & 2.2 \\
\hline a6 & 2 & 6 & 30 & -142 & 5.9 & 9.6 & 1.6 & 52.8 & 4.4 \\
\hline a7 & 2 & 6 & 40 & -141 & 6.1 & 11.1 & 1.8 & 56.6 & 2.2 \\
\hline a8 & 3 & 6 & 20 & -142 & 6.3 & 10.7 & 1.7 & 72.8 & 3.6 \\
\hline a9 & 4 & 6 & 20 & -140 & 6.7 & 12.5 & 1.8 & 72.7 & 3.8 \\
\hline al0 & 5 & 6 & 20 & -139 & 7.0 & 12.6 & 1.8 & 76.7 & 4.1 \\
\hline
\end{tabular}

${ }^{\mathrm{a}}$ Reaction temperature: $175^{\circ} \mathrm{C}$, nitrogen atmosphere; ${ }^{\mathrm{b}}$ mole ratio of $-\mathrm{NCO}$ to $-\mathrm{OH}$.

2.2. Synthesis of Polymer. The low molecular weight PLA prepolymer was synthesized through lactide ring-opening polymerization. The monomer (L-lactide) was first charged into the reactor. The catalyst solution was added by a syringe with capillary, and polymerization reaction was initiated. The reactor was heated with an oil bath while stirring, and through a period of $6 \mathrm{~h}$, the temperature was raised to $135^{\circ} \mathrm{C}$ while the pressure was reduced to $0.06 \mathrm{MPa}$. After the reaction, the polymers were dissolved by chloroform, precipitated by alcohol, and then dried under vacuum at $40^{\circ} \mathrm{C}$ for $48 \mathrm{~h}$.

After the ring-opening polymerization, the temperature was heightened to $175^{\circ} \mathrm{C}$ under nitrogen atmosphere, and calculated HDI was added to the reactor while stirring; the reaction was taken from 10 to $40 \mathrm{~min}$. After the reaction, the resulting polymer was dissolved in chloroform and precipitated in the excess of ethanol. The final product was isolated by filtration and dried in a vacuum at $40^{\circ} \mathrm{C}$ for $48 \mathrm{~h}$.

2.3. Characterizations. The number average molecular weight and weight average molecular weight of the PLA were determined by gel permeation chromatography (GPC) on a Waters 1515 HPLC system. Tetrahydrofuran was used as eluent at a flow rate of $1.0 \mathrm{~mL} \cdot \mathrm{min}^{-1}$. The temperature of the columns and detector was $40^{\circ} \mathrm{C}$. Calibrations were fulfilled with narrow molecular weight distributed polystyrene standards. Intrinsic viscosities were determined in chloroform at $(25 \pm 0.1)^{\circ} \mathrm{C}$ by Ubbelohde viscometer. $[\mathrm{y}]=\left[2 \times\left(\mathrm{\eta}_{\mathrm{sp}}-\ln \mathrm{\eta}_{r}\right)\right]^{0.5} / C=K M^{\alpha}, K=2.21 \times 10^{-4}$, $\alpha=0.77, \mathrm{\eta}_{r}=t / t_{0}, \mathrm{\eta}_{\mathrm{sp}}=1-\mathrm{\eta}_{r}$. Optical activities were tested in chloroform at $(25 \pm 0.1)^{\circ} \mathrm{C}$, by wzz-3 automatic polarimeter (Shanghai Precision Instrument Co.), and the measurement concentration was $9.0 \mathrm{mg} / \mathrm{mL}$. A Bruker VERTEX 70 FT-IR was used to scan the FTIT spectrum, and the ${ }^{1} \mathrm{H}-\mathrm{NMR}$ spectrum was recorded with a Bruker FT/AC-80 NMR spectrometer using $\mathrm{CDCl}_{3}$ as a solvent. DSC measurements were performed with a NETZSCH DSC-204F1 scanned at the rate of $10^{\circ} \mathrm{C} / \mathrm{min}$. A second-scan DSC spectrum was recorded after the sample was annealed to $0^{\circ} \mathrm{C}$. $X_{c}=\left(\Delta H_{m} / \Delta H_{m_{0}}\right) \times 100 \%, \Delta H_{m_{0}}=93 \mathrm{~J} / \mathrm{g}$ [16]. Xray diffraction patterns were recorded with a Bruker
ADVANCE-D8X X-ray diffractometer, scanned from $5^{\circ}$ to $40^{\circ}$.

\section{Results and Discussion}

3.1. Polymerization. The PLA prepolymer was synthesized using stannous octoate as the catalyst according to reaction [17]. After chain extending at $175^{\circ} \mathrm{C}$ for $10-40 \mathrm{~min}$, the reaction product was collected and analyzed. The analysis results were summarized in Table 1 . The molecular weight was increased several times after chain extension. It was suggested that HDI connects the hydroxyl end group so as to double grow the molecular weight. However, the increment of molecular weight was more than twice as shown in Table 1.

Table 1 shows the effect of the dosage of HDI, reaction time, and prepolymer molecular weight changes on the chain extension products. When the ratio of $[\mathrm{NCO}]$ to $[\mathrm{OH}]$ is 6 , after chain extending at $175^{\circ} \mathrm{C}$ for $20 \mathrm{~min}, M_{w}$ of 203,000 was obtained. From Run a1, a2, a3, and a4, with HDI increased, the molecular weight of PLA product growth multiples. While the amount of HDI was too much, molecular weight of products decreased. This is because the excessive HDI is unevenly distributed, and then crosslinking or branching happened, leading to the majority of low molecular weight prepolymer reacting with the remaining chain extender. When the ratio of $[\mathrm{NCO}]$ to $[\mathrm{OH}]$ is $8, M_{w}$ of 30000 was obtained and insoluble content reaching $3.9 \%$, indicate that the excess amount of the isocyanate group may also react with the other end of PLA molecule to form crosslink and branch structure according to reaction (3) of Figure 1. Run a3, a5, a6, and $\mathrm{a} 4$ indicated that long reaction time led to crosslinking, this was proved by the insoluble content. The molecular weight of the prepolymer changing in Run a3, a8, a9, and a10 shows that with increasing prepolymer molecular weight, the molecular weight of the chain-extended PLA increased, while insoluble content also increased. Optical activity test proved that the chain-extended product is laevorotary.

3.2. FT-IR and ${ }^{1} H-N M R$. Figure 2 shows the FT-IR spectrum of the PLA prepolymer and the chain-extended product 


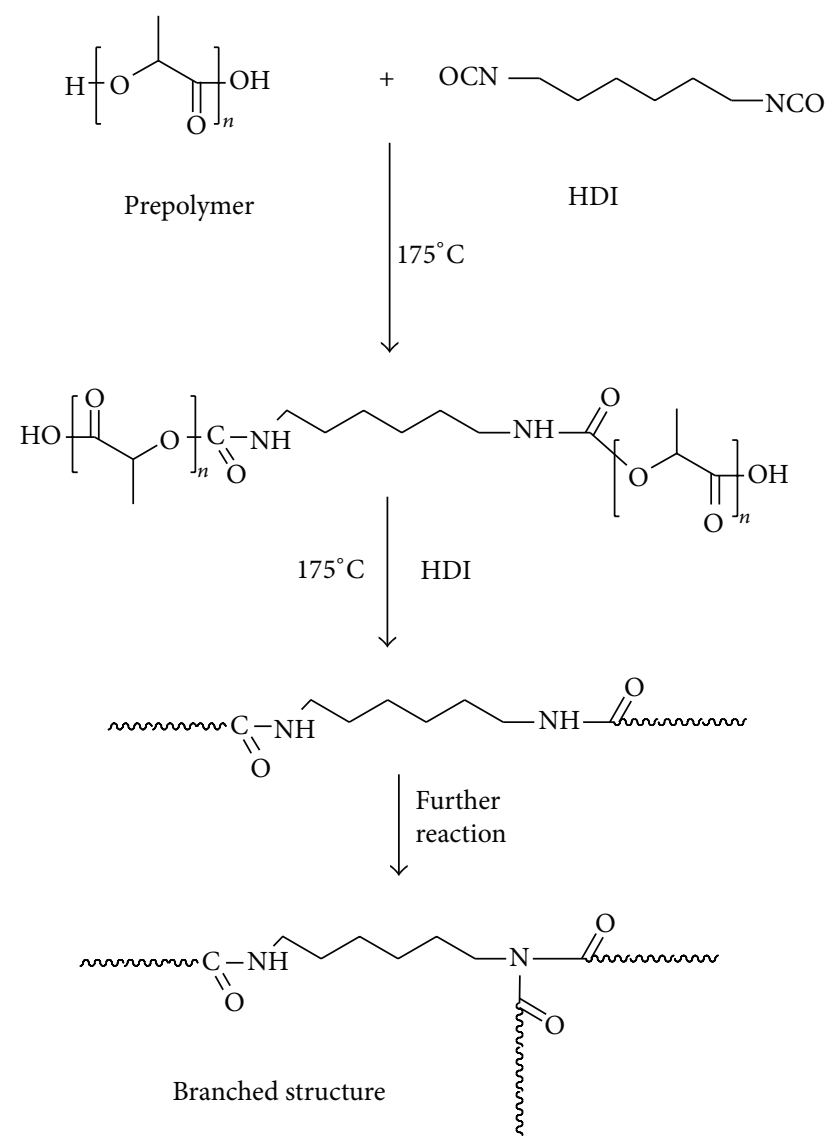

Figure 1: The synthesis scheme of the PLA chain extended with HDI.

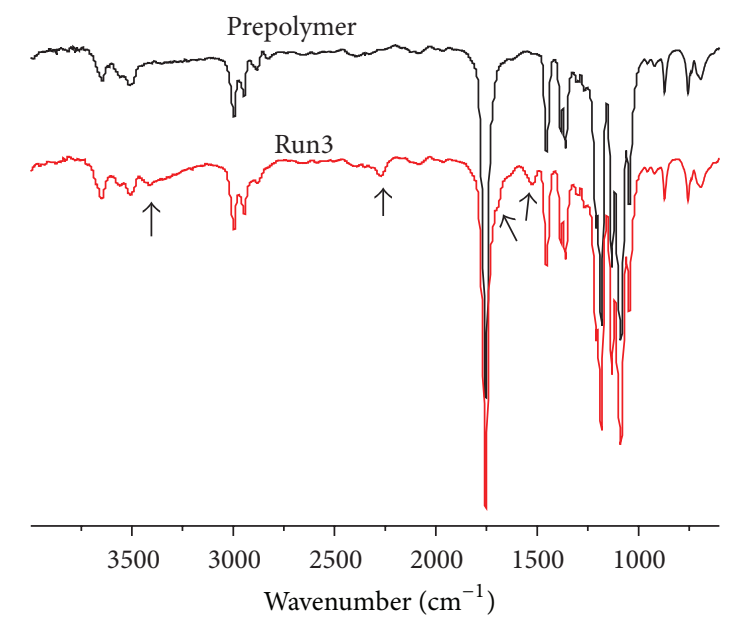

FIGURE 2: The infrared spectrum of the PLA prepolymer and the PLA chain extended with HDI (Run3).

with molar ratio at 6 of $\mathrm{NCO} / \mathrm{OH}$ after 20 min reaction. Both IR spectra have characteristic ester absorption bands at $1760 \mathrm{~cm}^{-1}$, but in the Run3, a shoulder peak appeared at $1691 \mathrm{~cm}^{-1}$ according to $-\mathrm{C}=\mathrm{O}$ adjoin to $-\mathrm{NH}$. The $-\mathrm{NH}$ flexural vibrations absorption peak at $1525 \mathrm{~cm}^{-1}$ and stretching vibrations absorption peak at $3413 \mathrm{~cm}^{-1}$ appeared after the chainextending reaction. The weak absorption at $720 \mathrm{~cm}^{-1}$ represents the $-\mathrm{CH}_{2}-$ from HDI. With the PLA crystallization peak at $756 \mathrm{~cm}^{-1}$, the peak strength weakened after the reaction [18].

Figure 3 shows the ${ }^{1} \mathrm{H}-\mathrm{NMR}\left(500 \mathrm{MHz}, \mathrm{TMS}, \mathrm{CDCl}_{3}\right)$ spectrum of the PLA prepolymer (Figure $3(\mathrm{a})$ ) and the PLA chain extended with HDI (Figure 3(b)). Both spectra exhibited the signal of methine group at $5.1 \mathrm{ppm}$ (a) and the signal of methyl group at $1.6 \mathrm{ppm}$ (c). The signal at $4.3 \mathrm{ppm}$ (b) in the spectrum shown in Figure 3(a) characteristic of the hydrogen of the methine next to a hydroxyl end group was found. But the signal at the same shift was not observed in the spectrum shown in Figure 3(b), because the HDI reacted with the hydroxyl group. The broad signals at $3.2 \mathrm{ppm}$ (b) and $4.1 \mathrm{ppm}(\mathrm{d})$ in the spectrum shown in Figure 3(b) were assigned to the unit of HDI in the PLA polymer chain [15], consistent with the results of FT-IR. The intensity ratio of the signal corresponding to urethane to the signal of HDI unit was not $1: 2$. This can be explained by the fact that the hydrogen in the urethane bond reacted with other polymer chain to form branch structure.

3.3. Gel Permeation Chromatography. Figure 4 shows the GPC spectra of the PLA chain extender with HDI. From the spectra we can comprehend that the distribution has two modes called a bimodal distribution. The characteristic peaks of low outflow time represent the high molecular weight chain-extending products, the peaks of high outflow time peak for the low molecular weight prepolymer. The chain extension reaction successfully happened in prepolymer, in the presence of HDI. With the increase of chain extender, reaction time, and prepolymer molecular weight, the high molecular weight part content increased in the products.

3.4. DSC. Table 2 shows the DSC thermograms of the PLA prepolymer and chain-extended PLA. After the chainextending reaction, the melt enthalpies were reduced. The $T_{g}$ and $T_{m}$ moved towards lower temperatures. This suggests that adding of chain extender had an effect on crystallinity of PLA according to the long chain alkane structure unit of the chain extender HDI. The crystallization of the chain-extended PLA is still above $40 \%$, yet crystal can be connected to each other, forming throughout the material of the continuous phase. So the highest used temperature can be increased close to the crystalline melting point [19].

Figure 5 shows the DSC thermogram of the PLA prepolymer and the PLA chain extended with different adding amount HDI. With the increasing amount of HDI, the crystallinity increased first and then decreased, indicating that a small amount of HDI was added to make the molecular chain growth, and also to maintain good crystallinity. Too much HDI makes the product of insoluble matter content increased, while it also reduces the product crystallinity.

Figure 6 shows the DSC thermograms with the time of the chain-extending reaction. When the reaction time was $30 \mathrm{~min}$, two obvious melting peaks of chain-extending product appeared, suggesting a phase transition process. The $T_{g}$ of the PLA chain extended with HDI decreased; it suggested 
TABLE 2: DSC data of the PLA prepolymer and the chain-extended PLA.

\begin{tabular}{|c|c|c|c|c|c|c|}
\hline Run & $M_{w}^{c}\left(\times 10^{-4} \mathrm{~g} / \mathrm{mol}\right)$ & Insoluble content (wt\%) & $\mathrm{T}_{g}\left({ }^{\circ} \mathrm{C}\right)$ & $T_{m}\left({ }^{\circ} \mathrm{C}\right)$ & $\Delta H_{m}(\mathrm{~J} / \mathrm{g})$ & $X_{c}(\%)$ \\
\hline Prepolymer & 2.0 & 0 & 59.0 & 170.3 & 52.9 & 56.8 \\
\hline 1 & 16.3 & 2.2 & 57.2 & 161.1 & 39.6 & 42.5 \\
\hline 2 & 12.1 & 3.0 & 57.2 & 164.5 & 41.1 & 44.1 \\
\hline 3 & 20.3 & 3.6 & 56.6 & 163.5 & 39.4 & 42.3 \\
\hline 6 & 9.6 & 4.4 & 55.5 & 158.8 & 42.2 & 45.3 \\
\hline 8 & 10.7 & 3.6 & 56.2 & 163.5 & 43.8 & 47.1 \\
\hline 9 & 12.5 & 3.8 & 55.8 & 164.1 & 43.5 & 46.7 \\
\hline 10 & 12.6 & 4.1 & 56.2 & 164.5 & 42.9 & 46.1 \\
\hline
\end{tabular}

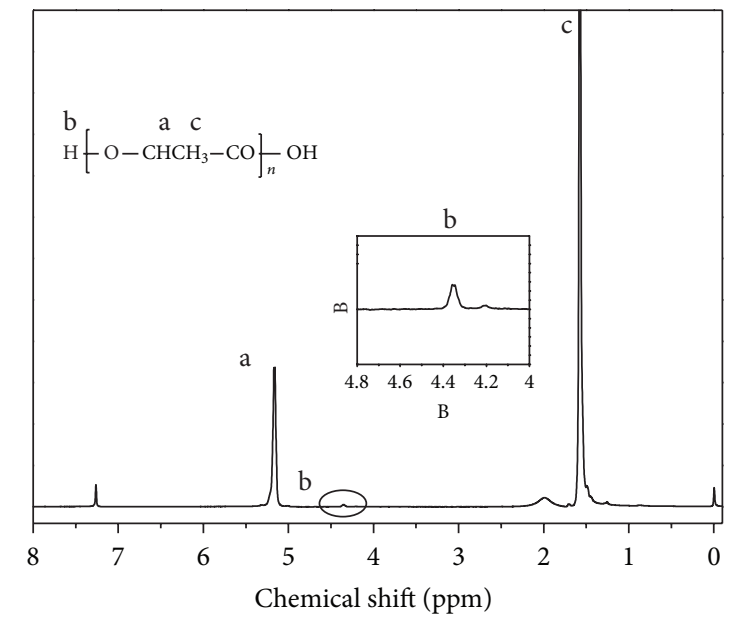

- Prepolymer

(a) prepolymer

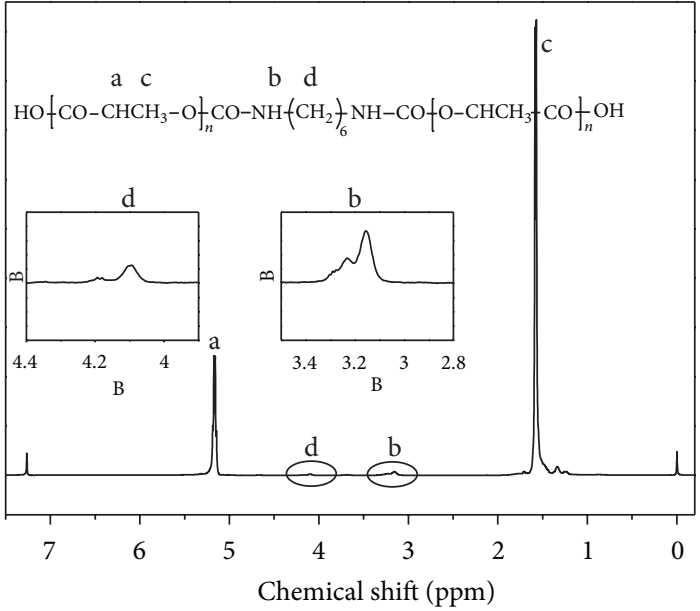

- Run3

b) Run3

Figure 3: ${ }^{1} \mathrm{H}-\mathrm{NMR}$ spectrum of PLA prepolymer (a) and the PLA chain extended with HDI (Run3) (b).

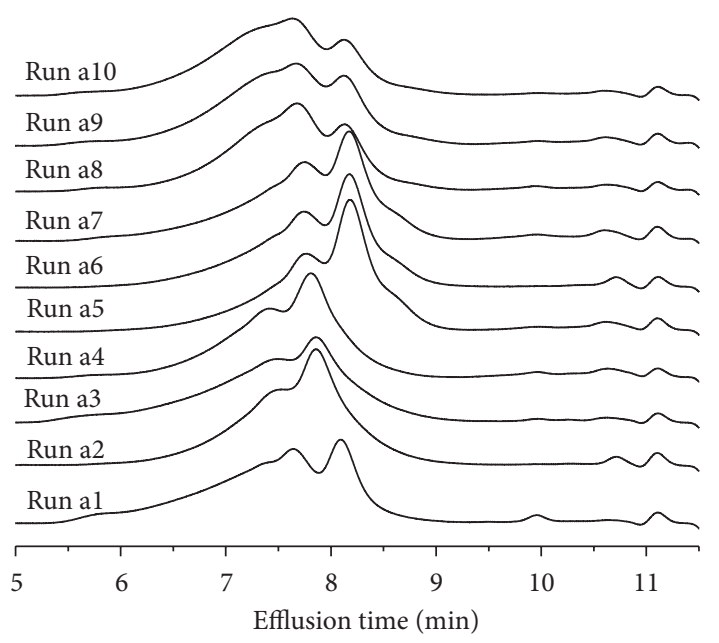

FIGURE 4: GPC spectrogram of the PLA chain extender with HDI.

that the longer reaction time resulted in insoluble matters increase. Because of the transition of crystal form crystalline part, the melting enthalpy increases and crystallinity of the PLA increased.

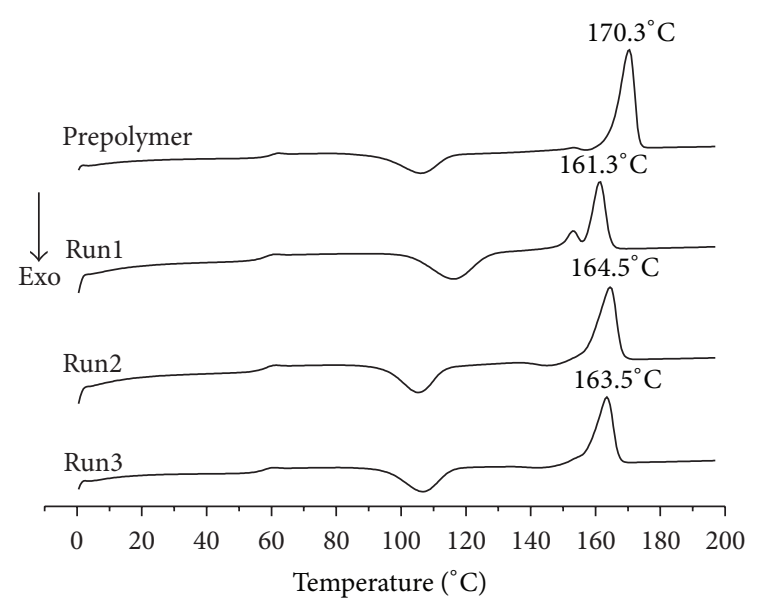

FIgURE 5: DSC thermogram of the prepolymer and the PLA chain extended with different adding amount HDI.

Figure 7 shows the DSC thermographs of the PLA from different molecular weight prepolymers. With the increase of molecular weight of PLA prepolymer, transformation of PLA crystal phenomenon gradually weakened and disappeared. 


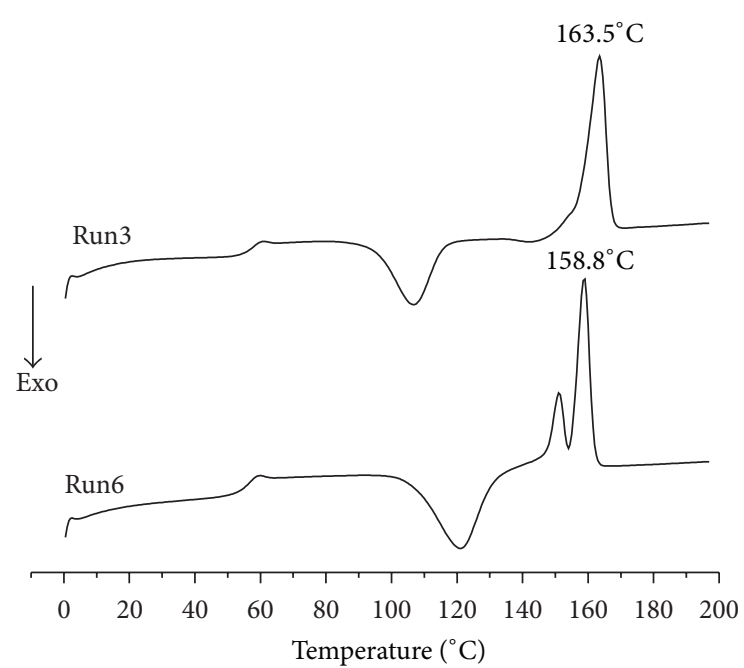

Figure 6: DSC thermogram of the chain-extended PLA with different chain-extending reaction time.

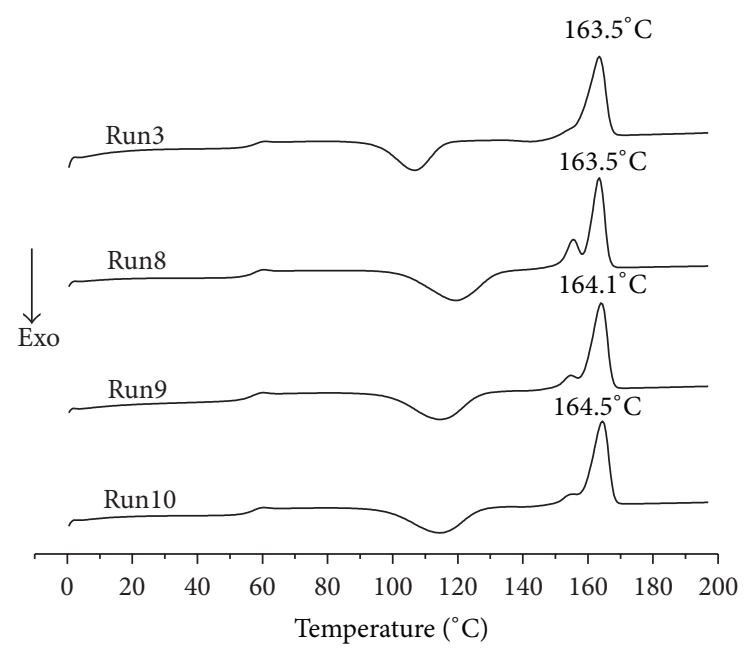

FIGURE 7: DSC thermogram of the PLA from different molecular weight prepolymers.

The insoluble matter content increased; the crystallinity decreased slightly.

3.5. X-Ray Diffraction. Figure 8 shows the X-ray diffraction pattern of the PLA prepolymer and the chain-extended PLA. The PLA prepolymer showed the sharp peak at $2 \theta$ about $16.5^{\circ}$ (020 reflection) and $18.8^{\circ}$ (023 reflection), while the peaks of the chain-extended samples were much lower and shifted the peaks at $2 \theta$ of $16.8^{\circ}$ and $19.1^{\circ}$. The results show that after the chain extender polymerization, the crystal type of the PLLA turns $\alpha^{\prime}$ to $\alpha$-crystal type [20,21], consistent with the results of DSC.

\section{Conclusions}

Higher molecular weight of poly(L-lactic acid) was prepared by using HDI chain-extending method with the low molecular weight PLA as the prepolymer. The weight average

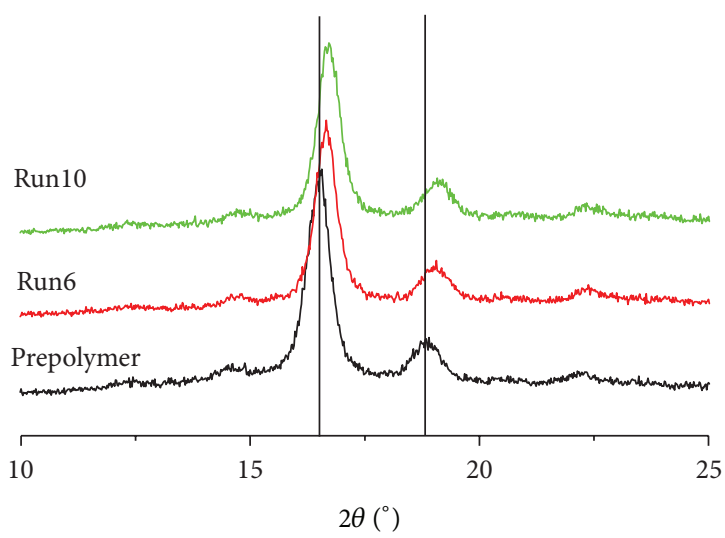

FIGURE 8: XRD pattern of the PLA prepolymer and the chainextended PLA.

molecular weight of the chain-extended PLA could reach up to $20.3 \times 10^{4} \mathrm{~g} / \mathrm{mol}$ by GPC measurement. Both FT-IR and ${ }^{1} \mathrm{H}-\mathrm{NMR}$ tests give a verification of structure, and the optically active characterized that the product was PLLA. Chain-extended PLLA had lower crystallinity by DSC and $\mathrm{X}$-ray diffraction because of the branched structure. The DSC and X-ray diffraction results both indicated that the crystalline of product changed from mixed $\alpha^{\prime}$ - and $\alpha$-crystal form to $\alpha$-crystal form.

\section{Acknowledgments}

This work was financially supported by the National Key Technology R\&D Program of China (2011BAE26B05), the National Nature Science Foundation of China (No. 21174074, 20774098, 51003050 and 51273100), Shandong Province Natural Science Fund for Distinguished Young Scholars (JQ201213) and the Nature Science Foundation of Shandong Province (ZR2011EMM008).

\section{References}

[1] L. Yu, K. Dean, and L. Li, "Polymer blends and composites from renewable resources," Progress in Polymer Science, vol. 31, no. 6, pp. 576-602, 2006.

[2] S. S. Ray and M. Bousmina, "Biodegradable polymers and their layered silicate nanocomposites: in greening the 21st century materials world," Progress in Materials Science, vol. 50, no. 8, pp. 962-1079, 2005.

[3] B. Gupta, N. Revagade, and J. Hilborn, "Poly(lactic acid) fiber: an overview," Progress in Polymer Science, vol. 32, no. 4, pp. 455482, 2007.

[4] J. Cheng, J. Sun, K. Wu et al., "Ring-opening polymerization of $\mathrm{D}$, L-lacide catalyzed with $\beta$-diketone compleses of Ti and Zr," Journal of Chemical Industry \& Engineering, vol. 27, no. 5, pp. 5-7, 2006.

[5] J. Shu, P. Wang, T. Zheng, L.-Y. Tian, and B.-X. Zhao, "Direct synthesis of ploy (L-lactic acid) by melt polycondensation," Material Science and Technology, vol. 15, no. 3, pp. 374-378, 2007.

[6] S. I. Woo, B. O. Kim, H. S. Jun, and H. N. Chang, "Polymerization of aqueous lactic acid to prepare high molecular weight 
poly(lactic acid) by chain-extending with hexamethylene diisocyanate," Polymer Bulletin, vol. 35, no. 4, pp. 415-421, 1995.

[7] Z. Wei, J. Ge, Z. Gu et al., "Study on biodegradable polymer materials based on poly(lactic acid) - I. Chain extending of low molecular weight Poly (lactic acid) with methylenediphenyl diisocyanate," Journal of Applied Polymer Science, vol. 74, pp. 2546-2551, 1999.

[8] J. Tuominen, J. Kylmä, and J. Seppälä, "Chain extending of lactic acid oligomers-2. Increase of molecular weight with 1,6hexamethylene diisocyanate and 2,2'-bis(2-oxazoline)," Polymer, vol. 43, no. 1, pp. 3-10, 2001.

[9] R. J. Feng and W. Z. Shi, "Influence of polymerization methods and chain-extension agent on relative molecular weight of polylactide," Petrochemical Technology, vol. 30, no. 2, pp. 103105, 2001.

[10] T. Yu, J. Ren, S. Gu, and M. Yang, "Preparation and characterization of biodegradable poly(lactic acid)-block-poly(eopencaprolactone) multiblock copolymer," Polymers for Advanced Technologies, vol. 21, no. 3, pp. 183-188, 2010.

[11] D. Cohn and A. Hotovely Salomon, "Designing biodegradable multiblock PCL/PLA thermoplastic elastomers," Biomaterials, vol. 26, no. 15, pp. 2297-2305, 2005.

[12] J.-B. Zeng, Y.-D. Li, W.-D. Li, K.-K. Yang, X.-L. Wang, and Y.Z. Wang, "Synthesis and properties of poly(ester urethane)s consisting of poly(l-lactic acid) and poly(ethylene succinate) segments," Industrial and Engineering Chemistry Research, vol. 48, no. 4, pp. 1706-1711, 2009.

[13] H. Li and M. A. Huneault, "Effect of chain extension on the properties of PLA/TPS blends," Journal of Applied Polymer Science, vol. 122, no. 1, pp. 134-141, 2011.

[14] B.-S. Park, J. C. Song, D. H. Park, and K.-B. Yoon, "PLA/chainextended PEG blends with improved ductility," Journal of Applied Polymer Science, vol. 123, no. 4, pp. 2360-2367, 2012.

[15] Z. Wang, Y. Zhao, and J. Wang, "Synthesis of polylactic acid biodegradable materials through chain extension," Chinese Journal of Synthetic Chemistry, vol. 11, pp. 106-110, 2012.

[16] M. Li, T. Jiao, Y . Wang et al., "Effect of plasticizer on crystallization morphology of biodegradable poly(lactic acid)," Plastic Science and Technology, vol. 39, no. 6, pp. 55-59, 2011.

[17] W.-W. Wang, Z. Yi, L. Jiang, and Y. Dan, "Synthesis of a polylactide macroinitiator via one-step polymerization," Polymeric Materials Science and Engineering, vol. 26, no. 1, pp. 12-15, 2010.

[18] Y. Hori, M. Suzuki, Y. Okeda et al., "A novel biodegradable poly(urethane ester) synthesized from poly(3-hydroxybutyrate) segments," Macromolecules, vol. 25, no. 19, pp. 5117-5118, 1992.

[19] M. He, Polymer Physics, Fudan University Publishing House, Shanghai, China, 2007.

[20] P. Pan, B. Zhu, W. Kai, T. Dong, and Y. Inoue, "Effect of crystallization temperature on crystal modifications and crystallization kinetics of poly(L-lactide)," Journal of Applied Polymer Science, vol. 107, no. 1, pp. 54-62, 2008.

[21] J. Zhang, K. Tashiro, H. Tsuji, and A. J. Domb, "Disorder-toorder phase transition and multiple melting behavior of poly(Llactide) investigated by simultaneous measurements of WAXD and DSC," Macromolecules, vol. 41, no. 4, pp. 1352-1357, 2008. 

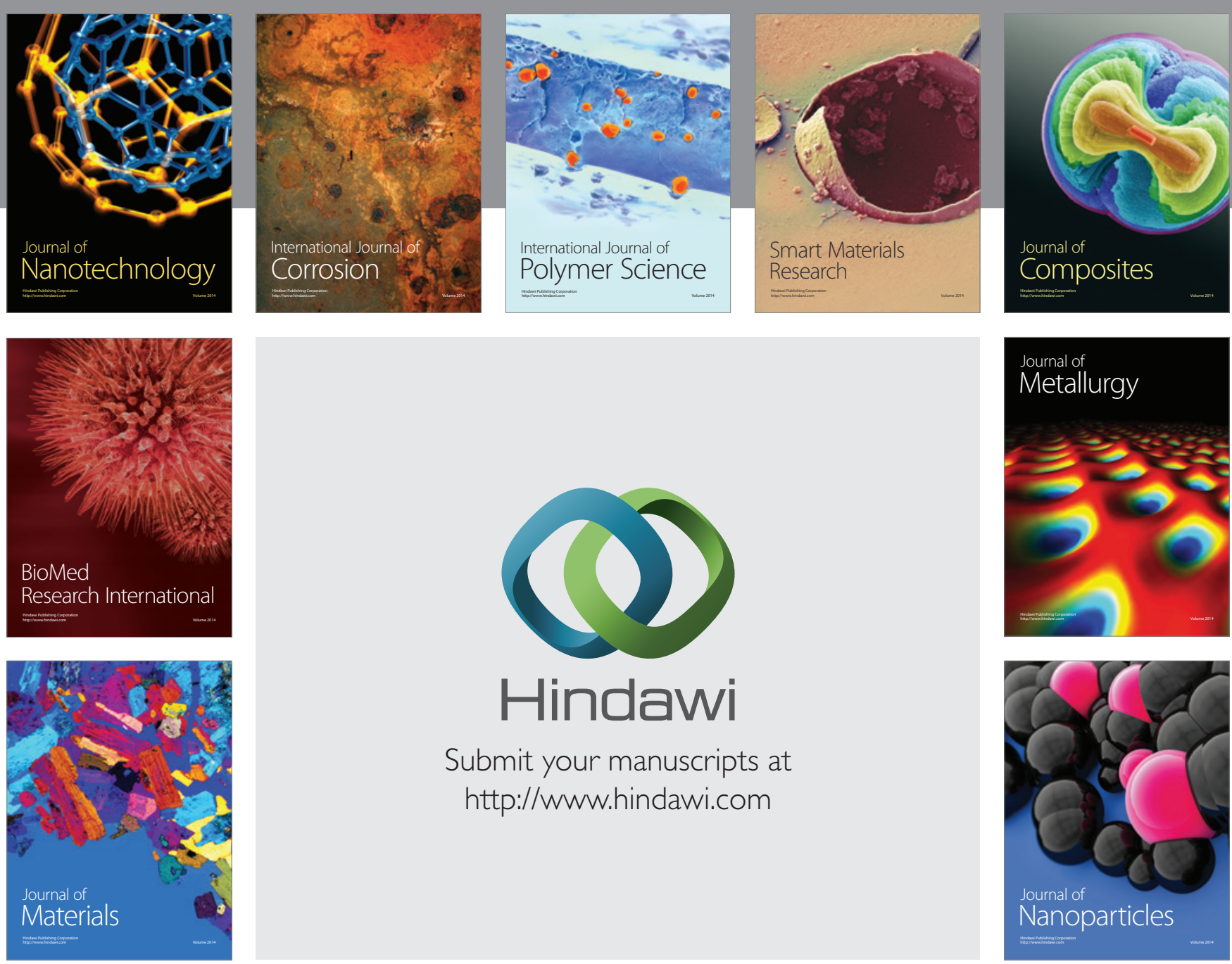

Submit your manuscripts at http://www.hindawi.com
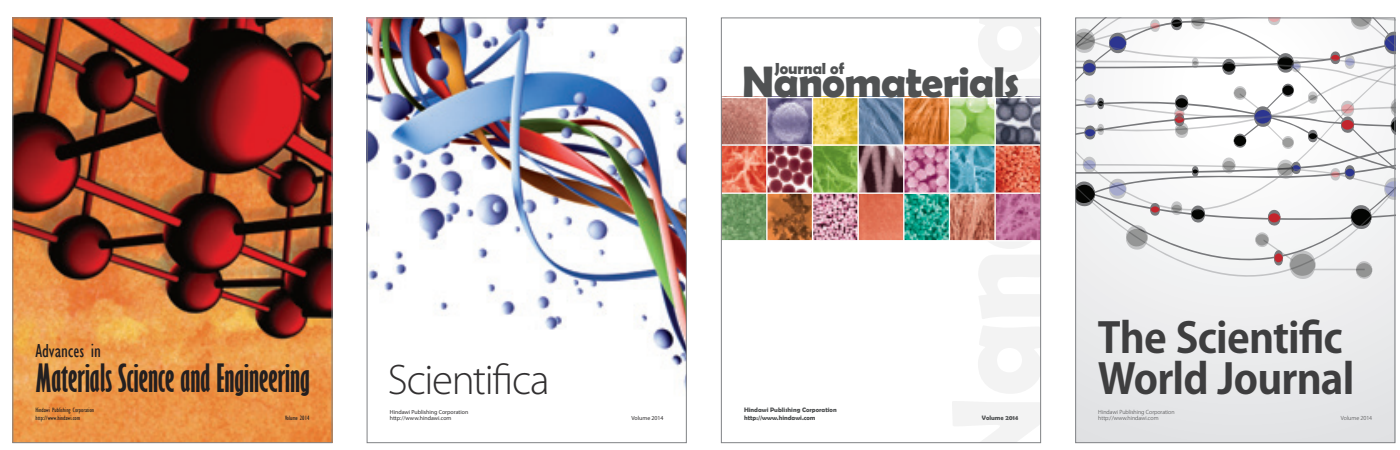

\section{The Scientific World Journal}
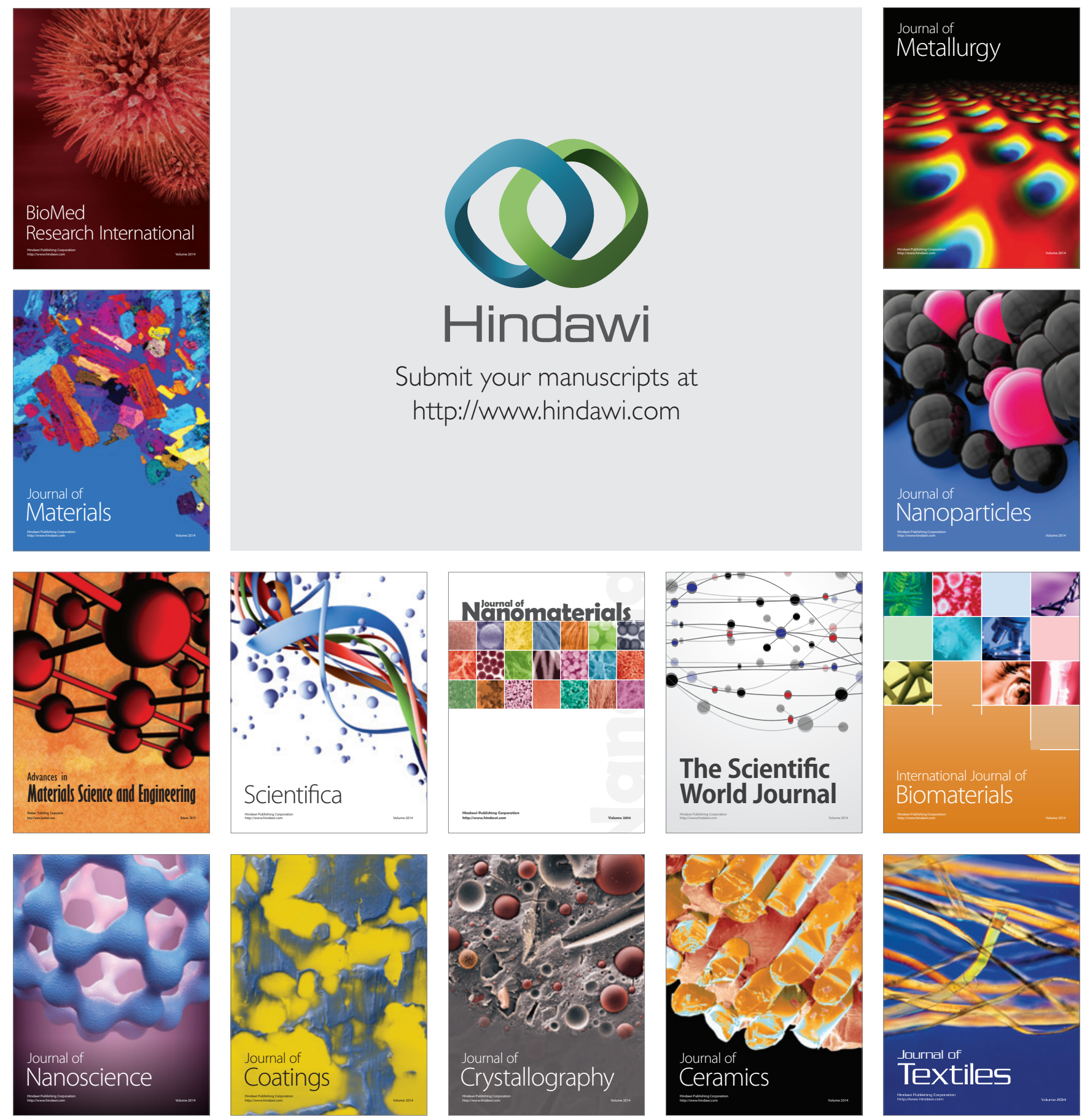